Research Article

\title{
Network Pharmacology-Based Prediction of Bioactive Compounds and Potential Targets of Wenjing Decoction for Treatment of Endometriosis
}

\author{
Yu-nan Liu $\mathbb{D}^{1},{ }^{1,2,3}$ Xiao-jing Hu ${ }^{(D},{ }^{3}$ Bei Liu $\mathbb{D}^{1,},{ }^{1,2}$ Yu-jie Shang $\mathbb{D}^{1,2}$ \\ Wen-ting $X u \mathbb{D}^{1,2}$ and Hui-fang Zhou $\mathbb{D}^{1,2}$ \\ ${ }^{1}$ Department of Gynecology, Affiliated Hospital of Nanjing University of Chinese Medicine, Nanjing, Jiangsu, China \\ ${ }^{2}$ Nanjing University of Chinese Medicine, Nanjing, Jiangsu, China \\ ${ }^{3}$ Department of Nephrology, The First Affiliated Hospital of Henan University of CM, Zhengzhou, Henan, China
}

Correspondence should be addressed to Hui-fang Zhou; 20183054@njucm.edu.cn

Received 26 April 2021; Accepted 14 June 2021; Published 25 June 2021

Academic Editor: Jianming GUO

Copyright (c) $2021 \mathrm{Yu}$-nan Liu et al. This is an open access article distributed under the Creative Commons Attribution License, which permits unrestricted use, distribution, and reproduction in any medium, provided the original work is properly cited.

Endometriosis is a chronic estrogen-dependent inflammatory disorder that negatively affects the quality of life in women. The Wenjing decoction (WJD) is a traditional Chinese medicine that has been shown to have a therapeutic effect on endometriosis. Our study systematically explored the mechanism of WJD against endometriosis using a network pharmacology approach. Potentially bioactive compounds of WJD and their possible targets were retrieved from the Traditional Chinese Medicine System Pharmacology Database and Analysis Platform. The protein-protein interaction network and herbs-compounds-genes multinetwork were constructed using Cytoscape for visualization. Subsequently, the signaling pathways of common targets were retrieved from the Kyoto Encyclopedia of Genes and Genomes (KEGG) databases, and molecular docking was performed using PyRx software. In total, 48 common targets were screened, such as IL6 and ESR1, which were related to inflammation and the endocrine system. The top five bioactive compounds were quercetin, kaempferol, wogonin, beta-sitosterol, and stigmasterol. KEGG enrichment analysis revealed 65 pathways containing inflammatory- and endocrine-related signaling pathways, such as the "TNF signaling pathway" and the "estrogen signaling pathway." Taken together, the results of our network pharmacology analysis predicted that certain active ingredients of WJD might treat endometriosis by regulating inflammation and/or endocrine, which provided references for further understanding and exploration of WJD on endometriosis.

\section{Introduction}

Endometriosis is one of the most common, benign gynecological disorders, affecting approximately $10 \%$ of women of reproductive age and $50 \%$ of those with fertility issues [1]. It is characterized by the growth of endometrial tissue (glands and stroma) outside the uterine cavity, most frequently on the ovaries, rectovaginal septum, and uterosacral ligaments [2]. Endometriosis, associated with pelvic inflammation, is most commonly manifested through dysmenorrhea, dyspareunia, gastrointestinal problems, deep pelvic pain, and infertility, which can negatively affect the quality of life among women $[3,4]$. Currently, hormonal therapy and surgery are the main courses to treat endometriosis [5]. Hormonal management can inhibit tissue growth, decrease damage, and reduce pain by stopping ovulation through the use of progestins, oral contraceptives, and gonadotropin-releasing hormone agonists [6]. Laparoscopic surgery is the gold standard for the diagnosis and treatment of endometriosis, and it can be used to distinguish the disease stage and remove endometriotic tissues, such as peritoneal implants, deep nodules, and ovarian cysts. However, both management strategies can only relieve the symptoms and delay recurrence, not cure the disease fundamentally, and even with several potential side effects. Thus, the clinical benefits are controversial [7]. 
In China, traditional Chinese medicine (TCM) is commonly used as an alternative therapy for endometriosis due to its significant therapeutic effect and lower toxicity $[8,9]$. Certain TCM formulas have been used to relieve endometriosis-associated pelvic inflammation and have achieved satisfying effects [10]. The Wenjing decoction (WJD), one of the most representative TCM prescriptions for endometriosis, has been used in Asian communities for more than 700 years. It consists of the following nine herbal materials: Radix Angelicae Sinensis (Danggui), Rhizoma Ligustici (Chuanxiong), Radix Ginseng (Renshen), Rhizoma Curcumae (Ezhu), Cortex Moutan (Mudanpi), Radix Achyranthis Bidentatae (Niuxi), Cortex Cinnamomi (Rougui), Radix Paeoniae Alba (Baishao), and Radix Glycyrrhizae (Gancao). The efficacy of WJD has been demonstrated in several animal and human studies, which showed that WJD could shrink existing endometriotic implants, alleviate dysmenorrhea, and increase the pregnancy rate. However, the mechanism and active ingredients of WJD have not yet been clarified [11-14].

Endometriosis is classified as an estrogen-dependent chronic inflammatory condition [15]. The local biosynthesis of estradiol by endometriotic focuses combined with inflammation in the peritoneal cavity develops an aberrant immune-endocrine microenvironment which is suitable for the growth and survival of ectopic lesions [1]. Estradiol then regulates downstream processes through nuclear estrogen receptors (ERs). Estradiol and ER signaling has been shown to be essential for lesion establishment in mouse models $[16,17]$. Moreover, Burns et al. demonstrated that ERs mediate the proliferation, adhesion, and angiogenesis of ectopic lesions [18]. Inflammation also has a main role in the progression of endometriosis. During endometriotic tissue formation, inflammatory cells are recruited to the lesions, which secrete multiple inflammatory factors, including interleukin 6 (IL6), IL8, and tumor necrosis factor $\alpha$ (TNF- $\alpha)$ [19-22]. In cases of endometriosis, these inflammatory factors not only fail to effectively remove endometrial debris from menstrual blood flow in the pelvic cavity but also facilitate their implantation, hemangiogenesis, and proliferation [23]. Considering the significance of inflammation and the endocrine system in endometriosis, we hypothesize that the bioactive compounds of WJD may have a therapeutic effect in endometriosis by regulating inflammation and/or the endocrine system.

Complex TCM formulations with dozens or even hundreds of different phytochemicals can act as multitarget therapies, which affect body function by potentially employing various mechanisms that have yet to be explored. In this study, we used network pharmacology to explore the possible bioactive compounds and molecular mechanisms of WJD employed in endometriosis treatment. These results could contribute to a better understanding of the associated molecular mechanisms employed by WJD against endometriosis.

\section{Materials and Methods}

2.1. Identification of Target Genes Linked to Selected WJD Compounds or Endometriosis. Information about the compounds from nine herbs and their protein targets was retrieved from the Traditional Chinese Medicine System Pharmacology Database and Analysis Platform (TCMSP, http://tcmspw.com/tcmsp.php). The drug feasibility of each candidate was assessed based on oral bioavailability (OB) and drug-likeness (DL) indices assigned from the TCMSP database. A high $O B$ value is a critical criterion for selecting biologically active molecules as candidates in drug development. DL assessments are used in drug design for determining chemical suitability and predicting pharmacodynamic and pharmacokinetic properties. In our study, molecules with $\mathrm{OB} \geq 30 \%$ and $\mathrm{DL} \geq 0.18$ were considered active compounds with potentially beneficial pharmacological effects. After obtaining these active compounds, we used the TCMSP database and the UniProt Knowledgebase (https://sparql.uniprot.org) to obtain the corresponding target protein and gene names, respectively.

A comprehensive list of endometriosis-related genes was obtained by searching public databases, including DisGeNet (https://www.disgenet.org), Therapeutic Target Database (TTD) (http://db.idrblab.net/ttd/), and DrugBank (https:// www.drugbank.ca). The overlapping genes between the bioactive compounds and the disease were identified and visualized using a Venn diagram.

2.2. Protein-Protein Interaction (PPI) and Multi-Network Construction and Kyoto Encyclopedia of Genes and Genomes (KEGG) Pathway Enrichment. The PPI network included information on the biological processes and molecular functions of cells. The identified overlapping target genes were introduced into the STRING network platform (https://string-db.org) with the "Homo sapiens" setting and the confidence score $>0.9$ to predict the interactions. The Cytoscape software 3.7.2 (http://www.cytoscape.org/) was used to visualize the network.

The symbols of herbs and compounds in WJD, as well as the endometriosis-associated targets, were uploaded into Cytoscape software for herbs-compounds-genes multinetwork construction. In the network, different shapes of the nodes represented potential genes, active compounds, or herbs. The nodes were evaluated based on the degree, which represented the number of edges between a single node and other network nodes. The importance of a node in the network was represented by the values of this indicator, and the higher the value, the greater the importance.

The KEGG database (http://www.genome.jp/kegg/) is suitable for large-scale systematic analysis of molecular networks with interacting genes. To assess a likely mechanism of WJD in the treatment of endometriosis, the KEGG pathway enrichment analysis was performed via DAVID Bioinformatics Resources 6.8 (https://david.ncifcrf.gov). 
After sorting the results according to the $P$ value, the top 30 significant pathway items were identified and presented via a bubble graph. Bigger and lower bubbles represented more significantly enriched pathway terms.

\subsection{Prediction of Molecular Docking between Bioactive} Compounds and Candidate Target Proteins. The bioactive composite crystal structures of the target proteins were retrieved from the Protein Data Bank (http://www.wwpdb. org), and the resulting macromolecule structure was preprocessed using PyRx 0.8 software. The molecular docking was achieved through Autodock Vina in the PyRx software. A semiempirical free energy calculation method was used to evaluate the receptor-ligand match.

2.4. Statistical Analysis. Fisher's exact test was used to identify significantly enriched KEGG signaling pathways. All the KEGG enrichment analysis results were sorted by $P<0.05$ as the critical criterion, and the first 30 pathways were screened out according to the $P$ value.

The flowchart of the network pharmacology approach used in this study is summarized in Figure 1.

\section{Results}

3.1. Bioactive Compounds and Targets of WJD. We identified 1,180 compounds of WJD in the TCMSP database, among which 171 met the screening criteria of $\mathrm{OB} \geq 30 \%$ and $\mathrm{DL} \geq 0.18$ (the compounds of Cortex Cinnamomi did not satisfy the filter criteria). After removing 18 repetitive compounds, we entered the remaining compounds into the TCMSP database and identified 272 nonrepetitive target points. To obtain the gene names of these proteins, we used the UniProt Knowledgebase for the conversion, and then we identified 269 target sites. The numbers of predicted ingredients, bioactive compounds, and corresponding targets are listed in Table 1.

\subsection{Endometriosis-Associated Target Genes and Bioactive} WJD Compounds. We retrieved 255 nonredundant endometriosis-related genes from the three databases (DisGeNet, TTD, and DrugBank) (Figure 2(a)). Mapping the endometriosis-related genes with the targets of bioactive compounds, we identified 48 intersected genes (Figure 2(b)). Detailed information about the bioactive compounds associated with the final target genes is provided in Table 2.

\subsection{Key Bioactive Compounds of WJD against Endometriosis.} The herbs-compounds-genes multinetwork included 8 herbs, 50 compounds, and 48 target genes (Figure 3 ). The distribution of the network connections indicated that WJD acted as a multitarget therapy against endometriosis by employing multiple bioactive compounds. Furthermore, we used the Cytoscape software to calculate the degree of the network. Seventeen compounds had a degree value exceeding the mean of all nodes (mean degree $=4.906$ ), thereby demonstrating their pharmacological importance. The following top five active compounds were selected: quercetin (MOL000098, degree=34), kaempferol (MOL000422, degree $=19)$, wogonin (MOL000173, degree =15), beta-sitosterol (MOL000358, degree =14), and stigmasterol (MOL00449, degree $=12$ ).

3.4. Key Hub Genes in the PPI Network. The PPI network was obtained from the STRING database and visualized by the Cytoscape software. There were 45 nodes and 366 edges representing the target genes and the interactions between the targets, respectively (Figure 4(a)). The mean degree value of all genes estimated was 16.267, and 26 targets exceeded the average, suggesting that WJD might exert its pharmacological effects mainly through these targets (Figure 4(b)). The top four target genes based on their degree values were TP53, IL6, VEGFA, and ESR1.

3.5. The KEGG Pathway Enrichment Analysis and the TargetPathway Network. Forty-eight potential targets of WJD in the treatment of endometriosis were uploaded to the DAVID database for enrichment analysis, and 65 pathways were obtained with $P<0.05$ (Figure 5(a)). The bubble diagram shown in Figure 5(b) represents the top 30 pathways, including the inflammatory- and endocrine-related signaling pathways, such as the "TNF signaling pathway" and the "estrogen signaling pathway."

3.6. Molecular Docking Predictions. In general, the binding energy between ligand and receptor is less than zero, indicating that they can bind spontaneously; generally, the lower the binding energy, the stronger the combined effect affinity. We selected the top 4 target genes (TP53, IL6, VEGFA, and ESR1) that encoded the following proteins: tumor protein p53 (TP53), IL6, vascular endothelial growth factor A (VEGF-A), and estrogen receptor alpha (ER $\alpha$ ). Using the selected proteins with the top 5 active compounds (quercetin, kaempferol, wogonin, beta-sitosterol, and stigmasterol), we performed molecular docking simulations. The docking results showed that these 4 targets could directly couple with the five bioactive compounds (Figure 6(a)). Furthermore, TP53 with quercetin, IL6 with wogonin, VEGF-A with wogonin, and $\mathrm{ER} \alpha$ with quercetin displayed the strongest combined effects, as shown by the specific docking diagrams in Figures 6(b)-6(e).

\section{Discussion}

Endometriosis is a chronic, estrogen-dependent, inflammatory disease. Several theories have been proposed for its pathogenesis, including the induction theory, Mayer's coelomic metaplasia theory, and the most widely accepted Sampson's implantation theory [24]. Sampson's theory claims that the ectopic endometrial foci originate from the exfoliation of endometrial cells during menstruation and spread to the peritoneum via the retrograde tubal flow [25]. Retrograde menstruation, as a major way of dissemination of endometriotic cells, occurs in almost all women, but only a 


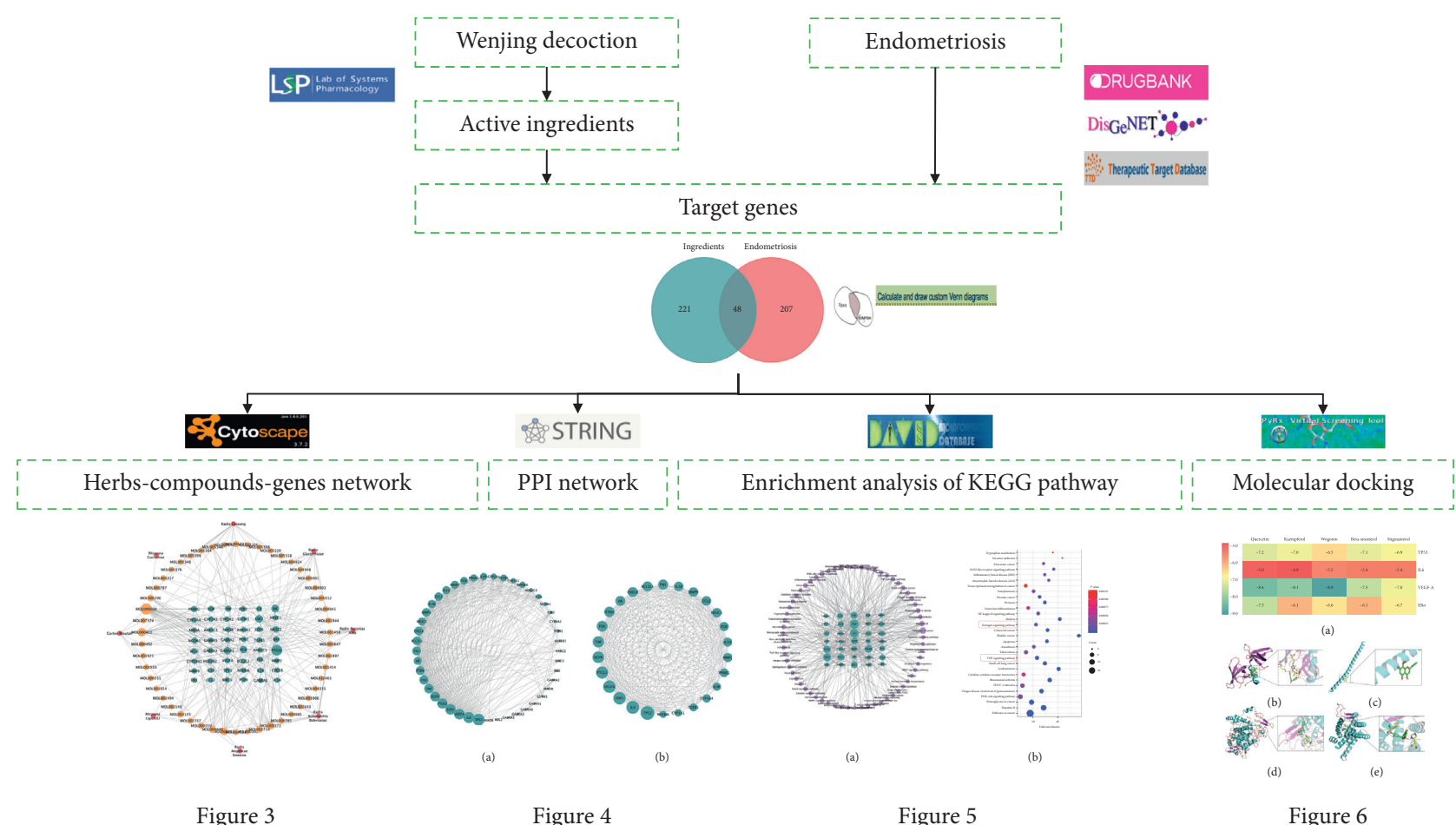

FIGURE 1: Flowchart of WJD treating endometriosis based on network pharmacology.

TABLE 1: Basic information about WJD compounds and predicted targets.

\begin{tabular}{lccc}
\hline Herbs & Number of compounds & Number of bioactive compounds & Number of predicted targets \\
\hline Radix Angelicae Sinensis & 105 & 2 & 54 \\
Rhizoma Ligustici & 108 & 7 & 30 \\
Radix Ginseng & 190 & 22 & 118 \\
Rhizoma Curcumae & 81 & 3 & 24 \\
Cortex Moutan & 55 & 11 & 173 \\
Radix Achyranthis Bidentatae & 176 & 20 & 187 \\
Cortex Cinnamomi & 100 & 0 & 0 \\
Radix Paeoniae Alba & 85 & 13 & 95 \\
Radix Glycyrrhizae & 280 & 93 & 238 \\
\hline
\end{tabular}

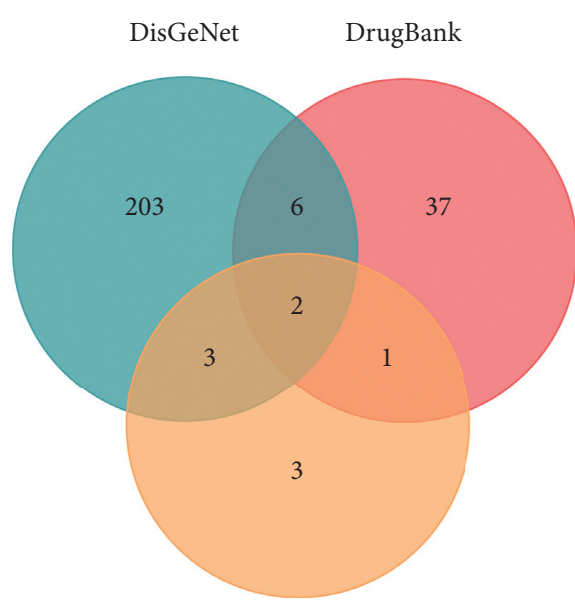

TTD

(a)

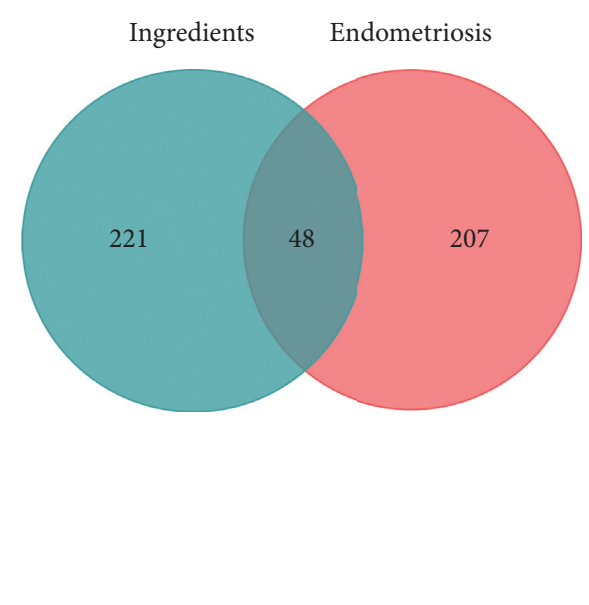

(b)

Figure 2: Venn diagrams. (a) Common genes from the three databases (DisGeNet, TTD, and DrugBank). (b) Overlapping target genes between WJD and endometriosis. 
TABLE 2: Information on the bioactive compounds of WJD.

\begin{tabular}{|c|c|c|c|c|c|c|}
\hline Number & Molecular ID & Active ingredients & $\begin{array}{l}\mathrm{OB} \\
(\%)\end{array}$ & $\mathrm{DL}$ & $\begin{array}{l}\text { Mapping target } \\
\text { number }\end{array}$ & Herbs \\
\hline 1 & MOL000358 & Beta-sitosterol & 36.91 & 0.75 & 10 & $\begin{array}{c}\text { Radix Angelicae Sinensis } \\
\text { Radix Paeoniae Alba } \\
\text { Radix Ginseng } \\
\text { Radix Achyranthis } \\
\text { Bidentatae }\end{array}$ \\
\hline 2 & MOL000449 & Stigmasterol & 43.83 & 0.76 & 8 & $\begin{array}{c}\text { Radix Angelicae Sinensis } \\
\text { Radix Ginseng } \\
\text { Radix Achyranthis } \\
\text { Bidentatae }\end{array}$ \\
\hline 3 & MOL000359 & Sitosterol & 36.91 & 0.75 & 2 & $\begin{array}{l}\text { Rhizoma Ligustici } \\
\text { Cortex Moutan } \\
\text { Radix Paeoniae Alba } \\
\text { Radix Glycyrrhizae }\end{array}$ \\
\hline 4 & MOL002157 & Wallichilide & 42.31 & 0.71 & 3 & Rhizoma Ligustici \\
\hline 5 & MOL002135 & Myricanone & 40.6 & 0.51 & 7 & Rhizoma Ligustici \\
\hline 6 & MOL002140 & Perlolyrine & 65.95 & 0.27 & 1 & Rhizoma Ligustici \\
\hline 7 & MOL001494 & Mandenol & 42 & 0.19 & 1 & Rhizoma Ligustici \\
\hline 8 & MOL001924 & Paeoniflorin & 53.87 & 0.79 & 2 & $\begin{array}{l}\text { Radix Paeoniae Alba } \\
\text { Radix Paeoniae Alba }\end{array}$ \\
\hline 9 & MOL000211 & Mairin & 55.38 & 0.78 & 1 & $\begin{array}{l}\text { Cortex Moutan } \\
\text { Radix Glycyrrhizae }\end{array}$ \\
\hline 10 & MOL001919 & Palbinone & 43.56 & 0.53 & 2 & Radix Paeoniae Alba \\
\hline 11 & MOL001925 & Paeoniflorin & 68.18 & 0.4 & 1 & $\begin{array}{l}\text { Radix Paeoniae Alba } \\
\text { Cortex Moutan }\end{array}$ \\
\hline 12 & MOL000492 & Cianidanol & 54.83 & 0.24 & 2 & $\begin{array}{l}\text { Radix Paeoniae Alba } \\
\quad \text { Cortex Moutan }\end{array}$ \\
\hline 13 & MOL000422 & Kaempferol & 41.88 & 0.24 & 14 & $\begin{array}{c}\text { Radix Paeoniae Alba } \\
\text { Cortex Moutan } \\
\text { Radix Ginseng } \\
\text { Radix Achyranthis } \\
\text { Bidentatae } \\
\text { Radix Glycyrrhizae }\end{array}$ \\
\hline 14 & MOL007374 & $\begin{array}{c}\text { 5-[[5-(4-Methoxyphenyl)-2-furyl }] \text { methylene }] \\
\text { barbituric acid }\end{array}$ & 43.44 & 0.3 & 1 & Cortex Moutan \\
\hline 15 & MOL000098 & Quercetin & 46.43 & 0.28 & 30 & $\begin{array}{c}\text { Cortex Moutan } \\
\text { Radix Achyranthis } \\
\text { Bidentatae } \\
\text { Radix Glycyrrhizae }\end{array}$ \\
\hline 16 & MOL000296 & Hederagenin & 36.91 & 0.75 & 7 & Rhizoma Curcumae \\
\hline 17 & MOL000787 & Fumarine & 59.26 & 0.83 & 2 & Radix Ginseng \\
\hline 18 & MOL005317 & Deoxyharringtonine & 39.27 & 0.81 & 2 & Radix Ginseng \\
\hline 19 & MOL005376 & Panaxadiol & 33.09 & 0.79 & 1 & Radix Ginseng \\
\hline 20 & MOL005348 & Ginsenoside-Rh4 & 31.11 & 0.78 & 1 & Radix Ginseng \\
\hline 21 & MOL005399 & Alexandrin & 36.91 & 0.75 & 9 & Radix Ginseng \\
\hline 22 & MOL005384 & Suchilactone & 57.52 & 0.56 & 10 & Radix Ginseng \\
\hline 23 & MOL005344 & Ginsenoside-Rh2 & 36.32 & 0.56 & 5 & Radix Ginseng \\
\hline 24 & MOL003648 & Inermin & 65.83 & 0.54 & 2 & Radix Ginseng \\
\hline 25 & MOL005321 & Frutinone A & 65.9 & 0.34 & 4 & Radix Ginseng \\
\hline 26 & MOL005356 & Girinimbin & 61.22 & 0.31 & 2 & Radix Ginseng \\
\hline 27 & MOL005308 & Aposiopolamine & 66.65 & 0.22 & 1 & Radix Ginseng \\
\hline 28 & MOL005320 & Arachidonate & 45.57 & 0.2 & 1 & Radix Ginseng \\
\hline 29 & MOL005318 & Dianthramine & 40.45 & 0.2 & 1 & Radix Ginseng \\
\hline 30 & MOL004924 & (-)-Medicocarpin & 40.99 & 0.95 & 1 & Radix Glycyrrhizae \\
\hline 31 & MOL004948 & Isoglycyrol & 44.7 & 0.84 & 3 & Radix Glycyrrhizae \\
\hline 32 & MOL005001 & Gancaonin $\mathrm{H}$ & 50.1 & 0.78 & 4 & Radix Glycyrrhizae \\
\hline 33 & MOL004903 & Liquiritin & 65.69 & 0.74 & 2 & Radix Glycyrrhizae \\
\hline 34 & MOL005012 & Licoagroisoflavone & 57.28 & 0.49 & 5 & Radix Glycyrrhizae \\
\hline 35 & MOL004941 & $\begin{array}{l}\text { (2R)-7-Hydroxy-2-(4-hydroxyphenyl)chroman- } \\
\text { 4-one }\end{array}$ & 71.12 & 0.18 & 4 & Radix Glycyrrhizae \\
\hline
\end{tabular}


TABLE 2: Continued.

\begin{tabular}{|c|c|c|c|c|c|c|}
\hline Number & Molecular ID & Active ingredients & $\begin{array}{l}\mathrm{OB} \\
(\%)\end{array}$ & $\mathrm{DL}$ & $\begin{array}{l}\text { Mapping target } \\
\text { number }\end{array}$ & Herbs \\
\hline 36 & MOL002844 & Pinocembrin & 64.72 & 0.18 & 4 & Radix Glycyrrhizae \\
\hline 37 & MOL000392 & Formononetin & 69.67 & 0.21 & 1 & Radix Glycyrrhizae \\
\hline 38 & MOL004328 & Naringenin & 59.29 & 0.21 & 2 & Radix Glycyrrhizae \\
\hline 39 & MOL001458 & Coptisine & 30.67 & 0.86 & 3 & $\begin{array}{c}\text { Radix Achyranthis } \\
\text { Bidentatae }\end{array}$ \\
\hline 40 & MOL003847 & Inophyllum E & 38.81 & 0.85 & 4 & $\begin{array}{c}\text { Radix Achyranthis } \\
\text { Bidentatae }\end{array}$ \\
\hline 41 & MOL002897 & Epiberberine & 43.09 & 0.78 & 3 & $\begin{array}{c}\text { Radix Achyranthis } \\
\text { Bidentatae }\end{array}$ \\
\hline 42 & MOL001454 & Berberine & 36.86 & 0.78 & 3 & $\begin{array}{c}\text { Radix Achyranthis } \\
\text { Bidentatae }\end{array}$ \\
\hline 43 & MOL012461 & 28-Norolean-17-en-3-ol & 35.93 & 0.78 & 1 & $\begin{array}{c}\text { Radix Achyranthis } \\
\text { Bidentatae }\end{array}$ \\
\hline 44 & MOL004355 & Spinasterol & 42.98 & 0.76 & 2 & $\begin{array}{c}\text { Radix Achyranthis } \\
\text { Bidentatae }\end{array}$ \\
\hline 45 & MOL001006 & Chondrillasterol & 42.98 & 0.76 & 2 & $\begin{array}{c}\text { Radix Achyranthis } \\
\text { Bidentatae }\end{array}$ \\
\hline 46 & MOL002643 & Delta 7-stigmastenol & 37.42 & 0.75 & 1 & $\begin{array}{c}\text { Radix Achyranthis } \\
\text { Bidentatae }\end{array}$ \\
\hline 47 & MOL000085 & Beta-daucosterol & 36.91 & 0.75 & 10 & $\begin{array}{c}\text { Radix Achyranthis } \\
\text { Bidentatae }\end{array}$ \\
\hline 48 & MOL000785 & Palmatine & 64.6 & 0.65 & 4 & $\begin{array}{c}\text { Radix Achyranthis } \\
\text { Bidentatae }\end{array}$ \\
\hline 49 & MOL000173 & Wogonin & 30.68 & 0.23 & 14 & $\begin{array}{c}\text { Radix Achyranthis } \\
\text { Bidentatae }\end{array}$ \\
\hline 50 & MOL002714 & Baicalein & 33.52 & 0.21 & 9 & $\begin{array}{c}\text { Radix Achyranthis } \\
\text { Bidentatae }\end{array}$ \\
\hline
\end{tabular}

proportion of them $(\sim 10 \%)$ develop endometriosis [7]. Hence, it appears that other factors may be involved in the development of ectopic endometriotic lesions.

Endometriosis has a distinct immune microenvironment and endocrine dysfunction, which promotes sustained proliferation, vascularization, and impaired apoptosis of the endometrial foci [26-28]. Our Venn diagram showed that the therapeutic effect of WJD on endometriosis was likely related to 48 genes (Figure 2). Furthermore, the results of the PPI network suggested that 26 targets, including IL6, ESR1, TP53, and VEGFA, were related to the occurrence and development of endometriosis, indicating that these targets might play an important role in the activity of WJD against endometriosis.

IL6, which is mainly secreted from macrophages and endometriotic cells, is involved in the inflammatory immune response. Moreover, it can inhibit apoptotic pathways, increase angiogenesis, that is, a process to develop new blood vessels and supply nutrients to growing ectopic tissues, and promote both cell adhesion and proliferation in endometriotic lesions [29]. $\mathrm{ER} \alpha$, an estrogen receptor, is encoded by ESR1. Not only have the levels of ER $\alpha$ been shown to be elevated in ectopic lesions, but they also promote the survival of cells in endometrial tissues by promoting cell proliferation, maintaining vascularization, and enabling cells to evade apoptosis [2]. TP53, a tumor suppressor, negatively regulates cell proliferation, inhibits angiogenesis, and induces apoptosis. In endometriotic tissues, reduction in the abundance and/or activity of TP53 frequently occurs, which promotes the uncontrolled proliferation and growth of ectopic lesions [30]. VEGF-A, a vital angiogenic factor expressed in ectopic tissues, mediates angiogenesis [31]. Based on the analysis of the top gene targets in the PPI network, we found that WJD might treat endometriosis by regulating inflammation and/or the endocrine system through IL6, ER $\alpha$, and other cytokines.

In this network pharmacology study, we established a herbs-compounds-genes multinetwork model using the Cytoscape software and identified 50 potentially bioactive compounds of WJD, including quercetin, kaempferol, wogonin, beta-sitosterol, and stigmasterol. Quercetin, an active ingredient common to Cortex Moutan, Radix Achyranthis Bidentatae, and Radix Glycyrrhizae, has been previously shown to inhibit cell proliferation, induce cell apoptosis, counteract inflammation, and regulate estrogen, as well as progesterone receptors on endometriosis autoimplanted mouse models [32, 33]. Kaempferol, presented in Radix Paeoniae Alba, Cortex Moutan, Radix Ginseng, Radix Achyranthis Bidentatae, and Radix Glycyrrhizae, exerts antiinflammatory effects by suppressing the release of IL6, IL1 $\beta$, IL18, and TNF- $\alpha$ [34]. It also has an antiangiogenic effect by reducing VEGF secretion and acts as an endocrine modulator to regulate steroid receptor expression in vivo $[35,36]$. Wogonin, an ingredient found in Radix Achyranthis Bidentatae, can decrease inflammatory and angiogenic hallmarks in a concentration-dependent manner [37]. Betasitosterol is a common compound in Radix Angelicae Sinensis, Radix Paeoniae Alba, Radix Ginseng, and Radix 


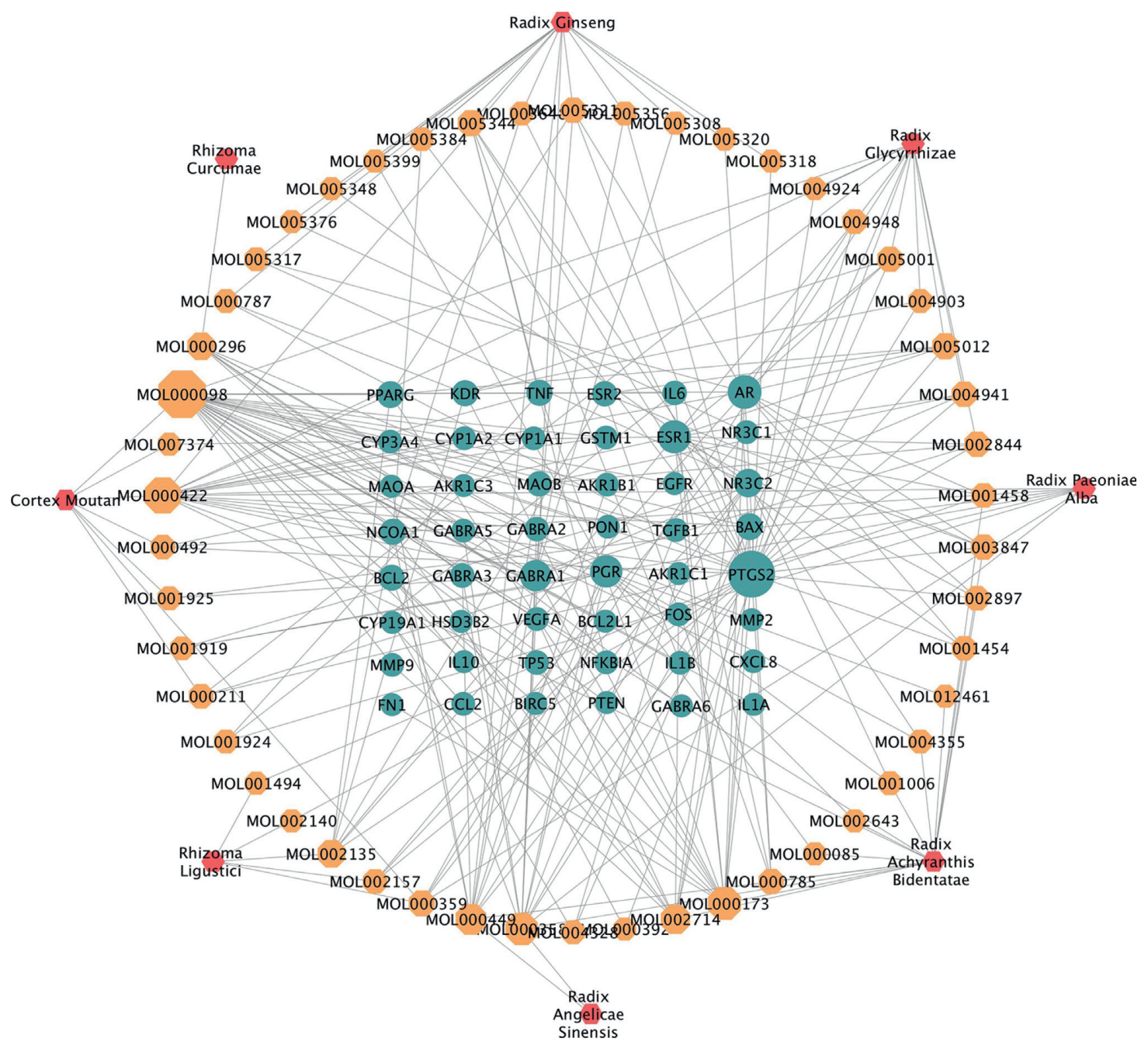

Figure 3: The herbs-compounds-genes multinetwork. The edges between nodes symbolize the interactions between them (more edges indicate greater relevance). Symbols: red hexagons, herbs; yellow octagons, bioactive compounds; blue circles, target genes.

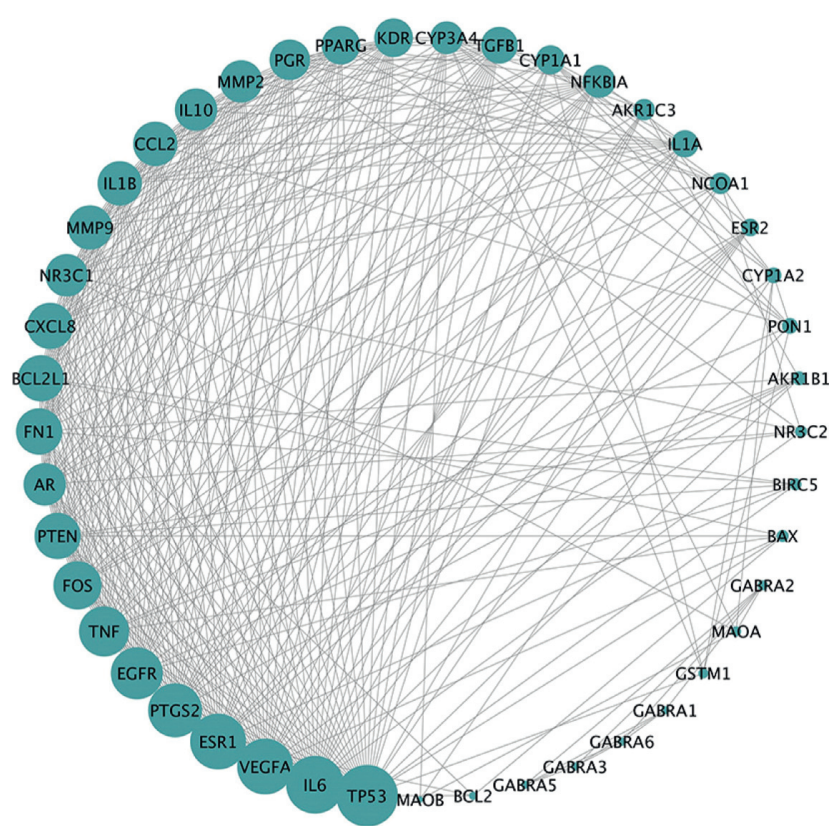

(a)

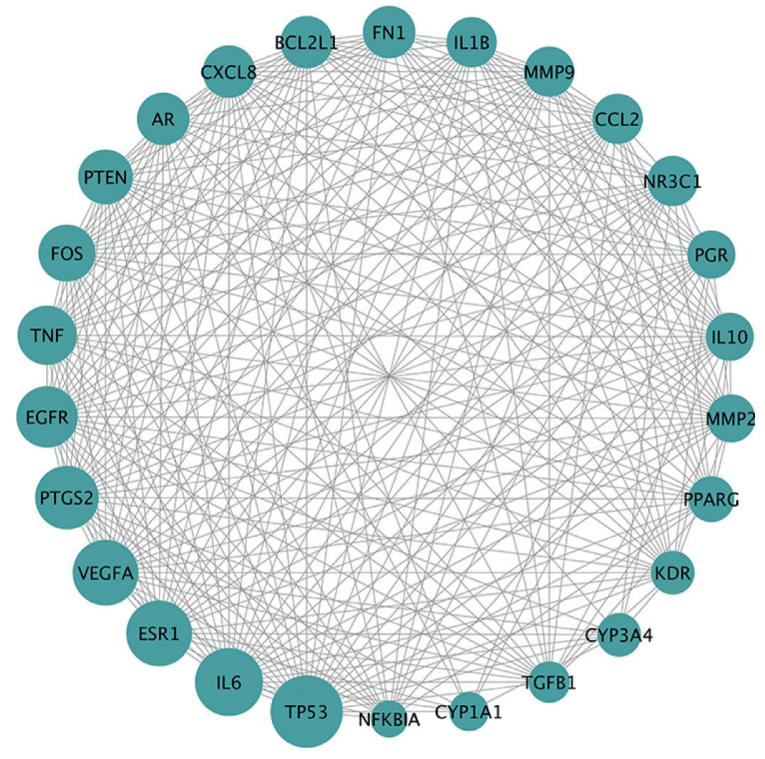

(b)

Figure 4: PPI network diagram of potential targets. (a) PPI network of 45 targets in the treatment of endometriosis using WJD. The blue circles mark the target proteins, and the node size corresponds to the degree value. (b) Diagram of 26 core targets. 


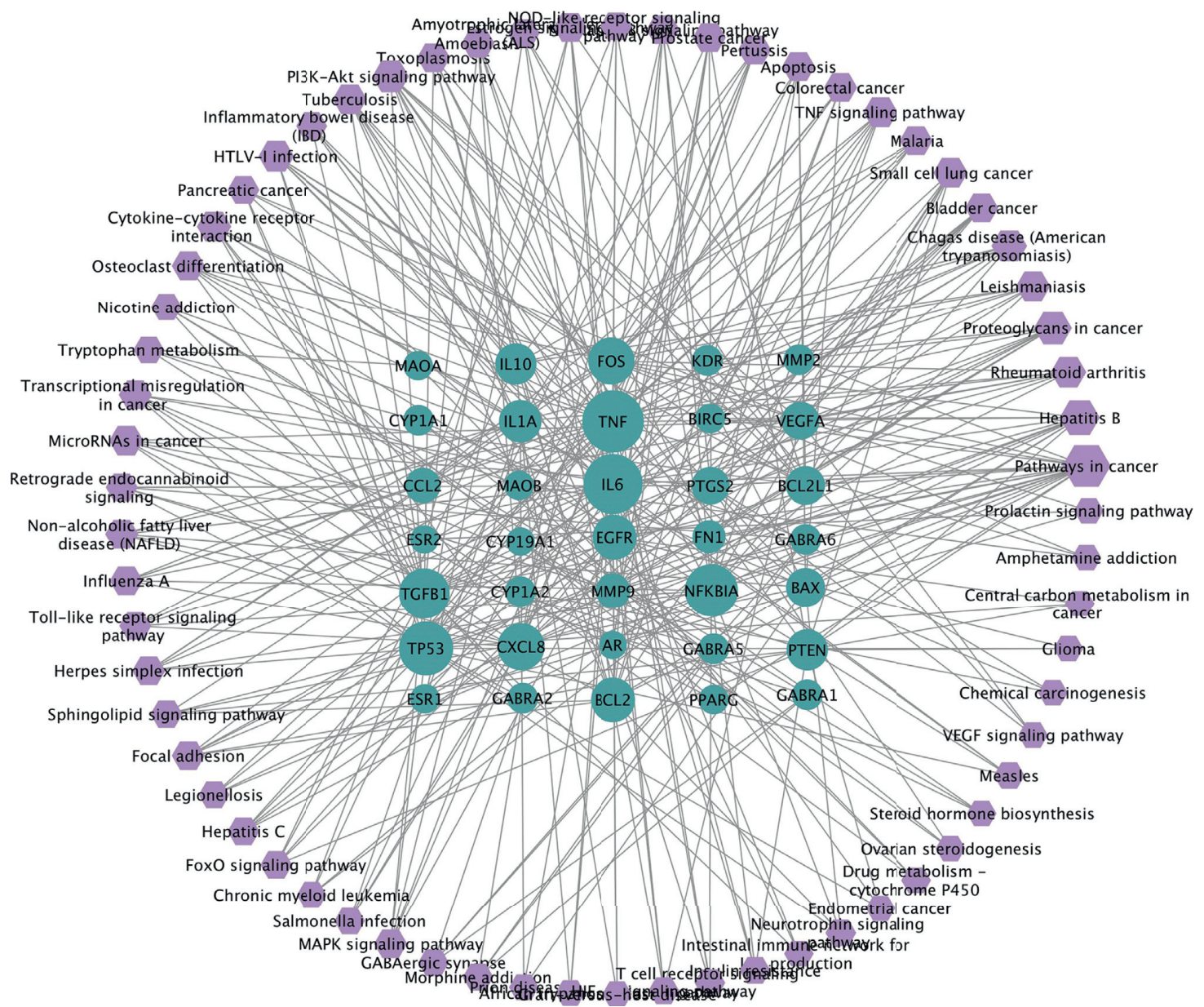

(a)

FIGURE 5: Continued. 


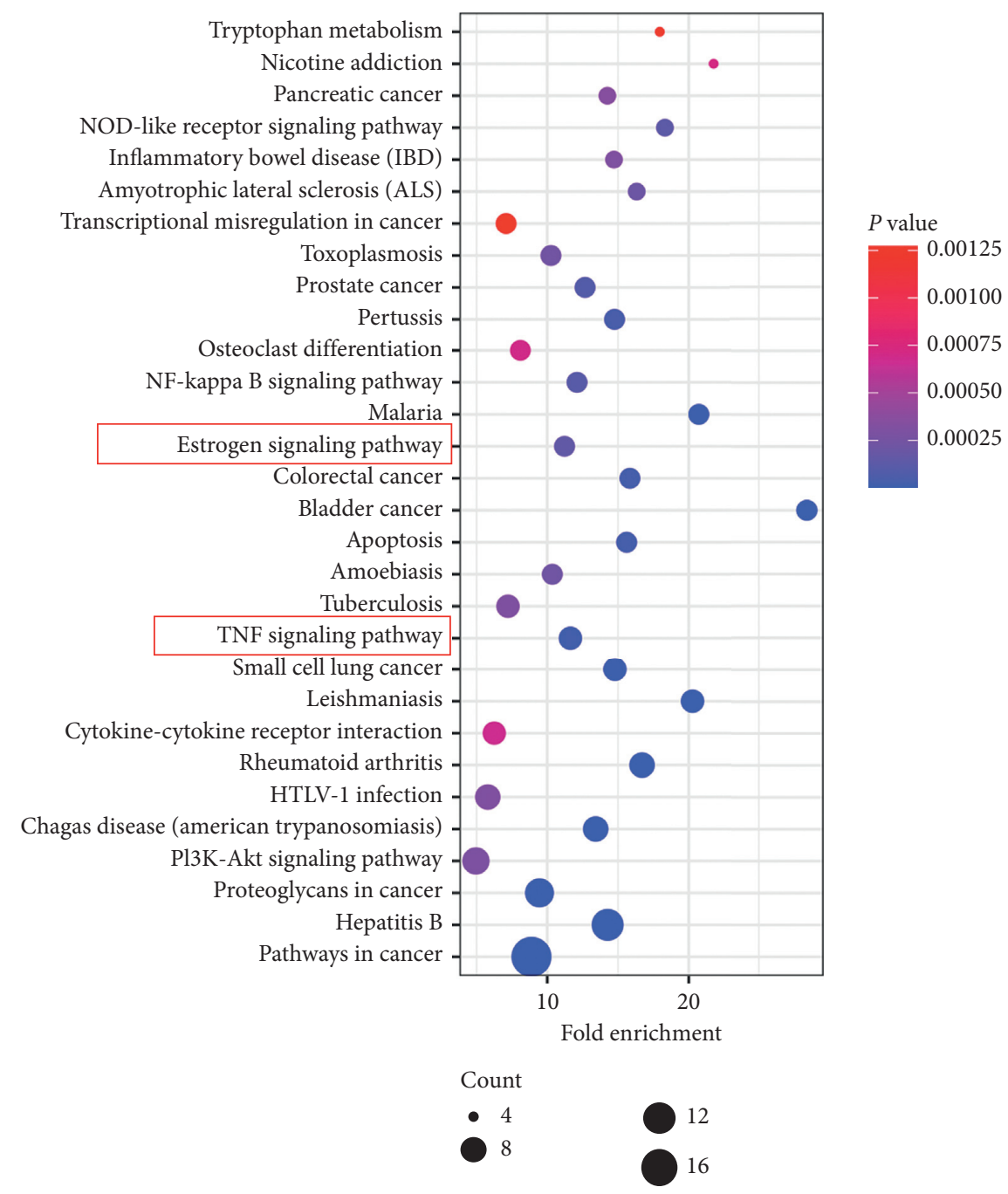

(b)

Figure 5: The KEGG signaling pathway diagrams. (a) The KEGG pathway enrichment analysis of 48 targets. Symbols: purple octagons, pathways; blue circles, targets. (b) Bubble diagram of the KEGG pathways. The $y$-axis shows the pathway name, and the $x$-axis indicates the percentage. The number of enriched genes in each pathway is presented via the bubble area, and the $P$ value is marked by the bubble color.

Achyranthis Bidentatae. In many in vitro and in vivo studies, it has been shown to possess various bioactivities, such as immunomodulatory, antimicrobial, and anti-inflammatory activities [38]. Stigmasterol, as a natural, plant-derived product with an anti-inflammatory effect, is found in Radix Angelicae Sinensis, Radix Ginseng, Radix Achyranthis Bidentatae, Rhizoma Ligustici, Cortex Moutan, Radix Paeoniae Alba, and Radix Glycyrrhizae. It has been reported that rats treated with stigmasterol have significantly suppressed the expression of proinflammatory mediators and increased the expression of anti-inflammatory cytokines [39]. Moreover, the molecular docking results showed that these five main compounds of WJD had a high binding affinity to the top four target proteins from the PPI network (Figure 6). Collectively, this network analysis suggested that the major active components of WJD may be effective for the treatment of endometriosis by counteracting inflammation and regulating the endocrine antiproliferation processes, along with other biological processes.

The results of the KEGG pathway analysis indicated that WJD might treat endometriosis via inflammatory- and endocrine-related signaling pathways, such as the "TNF signaling pathway" and the "estrogen signaling pathway." Concentrations of TNF are elevated in the peritoneal fluid and serum of endometriosis patients, especially in the early stages of the disease [40, 41]. An increased TNF concentration is associated with the enhanced motility of endometrial stromal cells through the regulation of ERK1/2 signaling, and it has been shown to activate systemic and local inflammation mechanisms in the development and progression of endometriosis by increasing chemokines and proinflammatory cytokines levels [42]. On the other hand, estrogen is a necessary hormone for the proliferation and expansion of ectopic lesions [43]. In a recent study, 

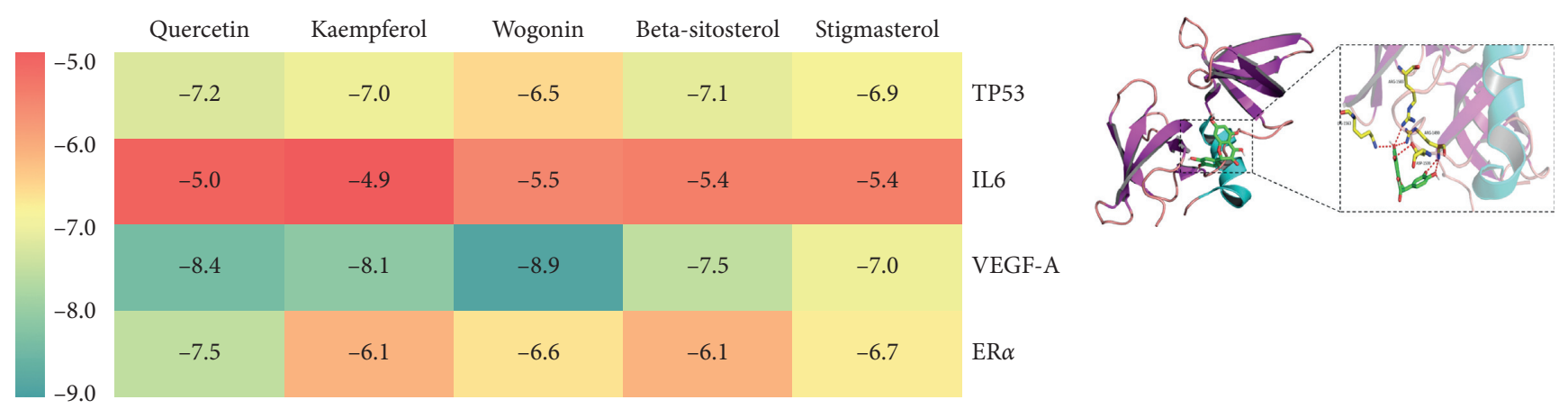

(a)

(b)

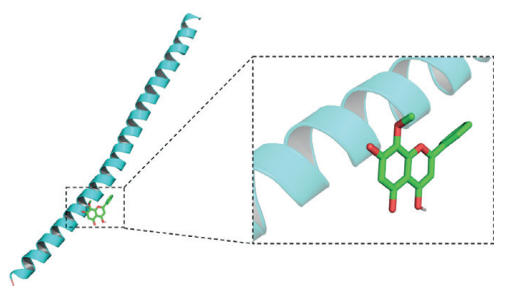

(c)

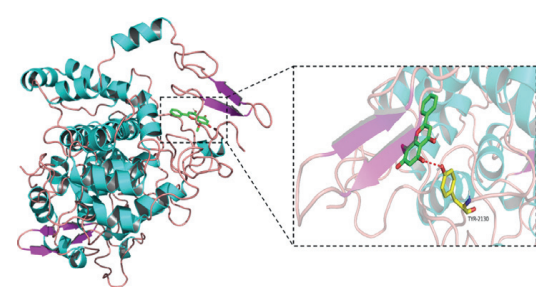

(d)

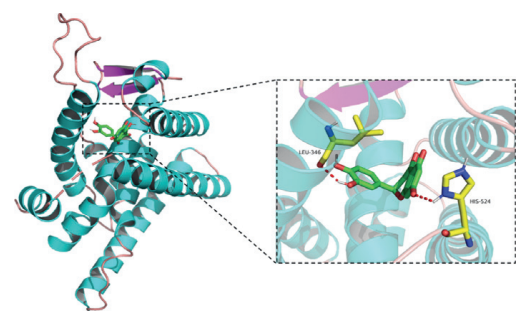

(e)

FIGURE 6: Molecular models of bioactive compounds binding to the predicted target proteins. (a) Heatmap of the binding energy by docking analysis. (b) The binding modes of TP53 and quercetin. (c) The binding modes of IL6 and wogonin. (d) The binding modes of VEGF-A and wogonin. (e) The binding modes of $\mathrm{ER} \alpha$ and quercetin.

treatment for endometriosis mainly blocks the production or function of estrogen [44]. ER $\alpha$ has a high affinity for estrogen, and its function is mainly mediated through ER $\alpha$, which is coupled with the "estrogen signaling pathway" [45].

In this study, network pharmacology was used to illustrate that WJD might treat endometriosis by regulating inflammation and/or the endocrine system. Our results provide guidance for further investigation of the mechanism, but there are still some limitations. Firstly, the acquisition of our active compounds and disease targets is based on existing databases that may not be comprehensive. For example, the active components of cinnamon in WJD did not meet the search filter criteria of the TCSMP database. Secondly, although we have identified possible related compounds and targets of WJD against endometriosis and performed molecular docking, it is still necessary to verify the involved mechanisms in vitro and in vivo.

\section{Conclusions}

In this study, we employed a network pharmacology-based approach to identify the bioactive compounds of WJD and their potential targets in endometriosis. The results suggested that the bioactive compounds of WJD against endometriosis included 48 targeted genes, among which IL6 and ESR1 were closely related to inflammation and the endocrine system, respectively. The mechanisms employed by WJD against endometriosis were related to 65 signaling pathways, including inflammatory- and endocrine-related signaling pathways, such as the "TNF signaling pathway" and the "estrogen signaling pathway." Thus, based on the network pharmacology analysis, we concluded that the mechanisms for treating endometriosis by WJD included the modulation of inflammation and/or the endocrine system, but in vitro and in vivo experimental validation is needed to corroborate our research further.

\section{Data Availability}

The data used to support the findings of this study are available from the corresponding author upon request.

\section{Conflicts of Interest}

The authors declare no conflicts of interest. 


\section{Authors' Contributions}

YL and XH contributed equally to this work. All authors were responsible for the study concept and design. Conceptualization was performed by $\mathrm{YL}, \mathrm{XH}$, and $\mathrm{HZ}$; methodology was carried out by YL and WX; software was provided by $\mathrm{XH}$ and $\mathrm{BL}$; formal analysis was conducted by $\mathrm{XH}$ and YS; data curation was performed by $\mathrm{YL}, \mathrm{XH}$, and $\mathrm{HZ}$; writing was done by YL and YS; funding acquisition was performed by HZ. All the authors approved the final paper.

\section{Acknowledgments}

This study was supported by grants from the National Natural Science Foundation of China (81973898 and 81774354), Major Project of Jiangsu Administration of Traditional Chinese Medicine Bureau (no. ZD201702), Leading Talents of Traditional Chinese Medicine in Jiangsu Province (SLJ0202), and Jiangsu Province Graduate Practice Innovation Program (SJCX20_0511).

\section{References}

[1] L. C. Giudice and L. C. Kao, "Endometriosis," The Lancet, vol. 364, no. 9447, pp. 1789-1799, 2004.

[2] K. T. Zondervan, C. M. Becker, K. Koga et al., "Endometriosis," Nature Reviews Disease Primers, vol. 4, no. 1, 2018.

[3] M. G. D. Broi, R. A. Ferriani, and P. A. Navarro, "Ethiopathogenic mechanisms of endometriosis-related infertility," JBRA Assisted Reproduction, vol. 23, no. 3, pp. 273-280, 2019.

[4] C. Tomassetti and T. D'Hooghe, "Endometriosis and infertility: insights into the causal link and management strategies," Best Practice \& Research Clinical Obstetrics \& Gynaecology, vol. 51, pp. 25-33, 2018.

[5] P. Vercellini, P. Viganò, E. Somigliana, and L. Fedele, "Endometriosis: pathogenesis and treatment," Nature Reviews Endocrinology, vol. 10, no. 5, pp. 261-275, 2014.

[6] S. Geoffron, G. Legendre, E. Darai, and N. Chabbert-Buffet, "Medical treatment of endometriosis: hormonal treatment of pain, impact on evolution and future perspectives," Presse Medical, vol. 46, no. 12, pp. 1199-1211, 2017.

[7] A. D. Greene, S. A. Lang, J. A. Kendziorski, J. M. Sroga-Rios, T. J. Herzog, and K. A. Burns, "Endometriosis: where are we and where are we going?" Reproduction, vol. 152, no. 3, pp. R63-R78, 2016.

[8] S. Z. Wu and H. Y. Xu, "Progress in the prevention and treatment of endometriosis recurrence by traditional Chinese and western medicine," Journal of Guangzhou University of TCM, vol. 34, no. 5, pp. 790-792, 2017.

[9] Z. X. Ni, W. Cheng, S. Sun, and C. Q. Yu, "Feasibility analysis of traditional Chinese medicine as the first line of conservative treatment for endometriosis," Maternal and Child Health Care of China, vol. 35, no. 01, pp. 178-181, 2020.

[10] W.-J. Zhou, H.-L. Yang, J. Shao et al., "Anti-inflammatory cytokines in endometriosis," Cellular and Molecular Life Sciences, vol. 76, no. 11, pp. 2111-2132, 2019.

[11] J. F. Lu, X. M. Cheng, J. Su, S. Lu, and B. Wang, "Effect of modified Wenjing Decoction on serum NO/ET-1 and TF/ TFPI in patients of endometriosis with cold syndrome," Chinese Journal of Integrative Medicine, vol. 40, no. 4, pp. 500-501, 2020.

[12] Y. Kang, M. H. Huang, H. P. Li, C. C. Zhang, and L. H. Qi, "Analysis of the effect of Wenjing Decoction on improving clinical symptoms and immune function of patients with endometriosis," Journal of Chinese Medicinal Materials, vol. 43, no. 2, pp. 482-485, 2020.

[13] H.-Y. Xu, Y.-Q. Zhang, Z.-M. Liu et al., "ETCM: an encyclopaedia of traditional Chinese medicine," Nucleic Acids Research, vol. 47, no. D1, pp. D976-D982, 2019.

[14] D. Wang, X. M. Cheng, X. H. Li et al., "Effect of modified Wenjing Decoction on the expression of Rho/ROCK signalling pathway in rats with syndrome of cold coagulation and blood stasis," Chinese Journal of Information on Traditional Chinese Medicine, vol. 27, no. 8, pp. 51-56, 2020.

[15] C. Hogg, A. W. Horne, and E. Greaves, "Endometriosis-associated macrophages: origin, phenotype, and function," Front Endocrinol (Lausanne), vol. 11, 2020.

[16] O. Bukulmez, D. B. Hardy, B. R. Carr, R. A. Word, and C. R. Mendelson, "Inflammatory status influences aromatase and steroid receptor expression in endometriosis," Endocrinology, vol. 149, no. 3, pp. 1190-1204, 2008.

[17] S. E. Bulun, D. Monsavais, M. E. Pavone et al., "Role of estrogen receptor-beta in endometriosis," Seminars in Reproductive Medicine, vol. 30, no. 1, pp. 39-45, 2012.

[18] K. A. Burns, K. F. Rodriguez, S. C. Hewitt, K. S. Janardhan, S. L. Young, and K. S. Korach, "Role of estrogen receptor signaling required for endometriosis-like lesion establishment in a mouse model," Endocrinology, vol. 153, no. 8, pp. 3960-3971, 2012.

[19] E. Barcz, Ł. Milewski, P. Dziunycz, P. Kamiński, R. Płoski, and J. Malejczyk, "Peritoneal cytokines and adhesion formation in endometriosis: an inverse association with vascular endothelial growth factor concentration," Fertility and Sterility, vol. 97, no. 6, pp. 1380-1386, 2012.

[20] Y.-Y. Fan, H.-Y. Chen, W. Chen, Y.-N. Liu, Y. Fu, and L.-N. Wang, "Expression of inflammatory cytokines in serum and peritoneal fluid from patients with different stages of endometriosis," Gynecological Endocrinology, vol. 34, no. 6, pp. 507-512, 2018.

[21] J. Sikora, M. Smycz-Kubanska, A. Mielczarek-Palacz, and Z. Kondera-Anasz, "Abnormal peritoneal regulation of chemokine activation-the role of IL-8 in pathogenesis of endometriosis," American Journal of Reproductive Immunology, vol. 77, no. 4, 2017.

[22] J. Sikora, A. Mielczarek-Palacz, and Z. Kondera-Anasz, "Association of the precursor of interleukin- $1 \beta$ and peritoneal inflammation-role in pathogenesis of endometriosis," Journal of Clinical Laboratory Analysis, vol. 30, no. 6, pp. 831-837, 2016.

[23] T. Zhang, C. De Carolis, G. C. W. Man, and C. C. Wang, "The link between immunity, autoimmunity and endometriosis: a literature update," Autoimmunity Reviews, vol. 17, no. 10, pp. 945-955, 2018.

[24] D. Vinatier, G. Orazi, M. Cosson, and P. Dufour, "Theories of endometriosis," European Journal of Obstetrics \& Gynecology and Reproductive Biology, vol. 96, no. 1, pp. 21-34, 2001.

[25] J. A. Sampson, "Metastatic or embolic endometriosis, due to the menstrual dissemination of endometrial tissue into the venous circulation," American Journal of Pathology, vol. 3, no. 2, pp. 93-110, 1927.

[26] M. C. Johnson, M. Torres, A. Alves et al., “Augmented cell survival in eutopic endometrium from women with endometriosis: expression of c-myc, TGF-betal and bax genes," Reproductive Biology and Endocrinology, vol. 3, no. 1, p. 45, 2005.

[27] S. Suryawanshi, X. Huang, E. Elishaev et al., "Complement pathway is frequently altered in endometriosis and 
endometriosis-associated ovarian cancer," Clinical Cancer Research, vol. 20, no. 23, pp. 6163-6174, 2014.

[28] E. Chantalat, M. C. Valera, C. Vaysse et al., "Estrogen receptors and endometriosis," International Journal of Molecular Sciences, vol. 21, no. 8, 2020.

[29] Y.-J. Kang, I. C. Jeung, A. Park et al., "An increased level of IL6 suppresses NK cell activity in peritoneal fluid of patients with endometriosis via regulation of SHP-2 expression," Human Reproduction, vol. 29, no. 10, pp. 2176-2189, 2014.

[30] S. J. Han and B. W. O'Malley, “The dynamics of nuclear receptors and nuclear receptor coregulators in the pathogenesis of endometriosis," Human Reproduction Update, vol. 20, no. 4, pp. 467-484, 2014.

[31] M. W. Laschke and M. D. Menger, "Anti-angiogenic treatment strategies for the therapy of endometriosis," Human Reproduction Update, vol. 18, no. 6, pp. 682-702, 2012.

[32] S. Park, W. Lim, F. W. Bazer, K.-Y. Whang, and G. Song, "Quercetin inhibits proliferation of endometriosis regulating cyclin D1 and its target microRNAs in vitro and in vivo," The Journal of Nutritional Biochemistry, vol. 63, pp. 87-100, 2019.

[33] Y. Cao, M. F. Zhuang, Y. Yang et al., "Preliminary study of quercetin affecting the hypothalamic-pituitary-gonadal axis on rat endometriosis model," Evidence-based Complementary and Alternative Medicine: eCAM, vol. 2014, Article ID 781684, 12 pages, 2014.

[34] X.-L. Tang, J.-X. Liu, W. Dong et al., "Protective effect of kaempferol on LPS plus ATP-induced inflammatory response in cardiac fibroblasts," Inflammation, vol. 38, no. 1, pp. 94-101, 2015.

[35] M. Ilhan, Z. Ali, I. A. Khan, H. Taştan, and E. Küpeli Akkol, "The regression of endometriosis with glycosylated flavonoids isolated from Melilotus officinalis (L.) Pall. in an endometriosis rat model," Taiwanese Journal of Obstetrics and Gynecology, vol. 59, no. 2, pp. 211-219, 2020.

[36] M. F. Toh, E. Mendonca, S. L. Eddie et al., "Kaempferol exhibits progestogenic effects in ovariectomized rats," Journal of Steroids \& Hormonal Science, vol. 5, no. 3, p. 136, 2014.

[37] G. Gong, H. Wang, X. Kong, R. Duan, T. T. X. Dong, and K. W. K. Tsim, "Flavonoids are identified from the extract of Scutellariae Radix to suppress inflammatory-induced angiogenic responses in cultured RAW 264.7 macrophages," Scientific Reports, vol. 8, no. 1, p. 17412, 2018.

[38] S. Babu and S. Jayaraman, "An update on $\beta$-sitosterol: a potential herbal nutraceutical for diabetic management," Biomedicine \& Pharmacotherapy, vol. 131, Article ID 110702, 2020.

[39] M. Ahmad Khan, A. H. M. G. Sarwar, R. Rahat, R. S. Ahmed, and S. Umar, "Stigmasterol protects rats from collagen induced arthritis by inhibiting proinflammatory cytokines," International Immunopharmacology, vol. 85, Article ID $106642,2020$.

[40] A. Olivera, M. A. Beaven, and D. D. Metcalfe, "Mast cells signal their importance in health and disease," Journal of Allergy and Clinical Immunology, vol. 142, no. 2, pp. 381-393, 2018.

[41] F. M. Howard, "Endometriosis and mechanisms of pelvic pain," Journal of Minimally Invasive Gynecology, vol. 16, no. 5, pp. 540-550, 2009.

[42] Y. Peng, J. Ma, and J. Lin, "Activation of the CXCL16/CXCR6 Axis by TNF- $\alpha$ contributes to ectopic endometrial stromal cells migration and invasion," Reproductive Sciences, vol. 26, no. 3, pp. 420-427, 2019.

[43] I. Paterni, C. Granchi, J. A. Katzenellenbogen, and F. Minutolo, "Estrogen receptors alpha $(\mathrm{ER} \alpha)$ and beta $(\mathrm{ER} \beta)$ : subtype-selective ligands and clinical potential," Steroids, vol. 90, pp. 13-29, 2014.

[44] B. C. Parente, D. S. A. Bentes, B. Bianco, and D. M. Christofolini, "The effect of hormones on endometriosis development," Minerva Obstetrics and Gynecology, vol. 63, no. 4, pp. 375-386, 2011.

[45] S. Asghari, A. Valizadeh, L. Aghebati-Maleki, M. Nouri, and M. Yousefi, "Endometriosis: perspective, lights, and shadows of etiology," Biomedicine \& Pharmacotherapy, vol. 106, pp. 163-174, 2018. 